\title{
Cascade Control for Hydraulic Automatic Gauge Control of Hot Rolling Mills Based on Data-driven Theory
}

\author{
Xiaodong Zhang, Xinping Liu \\ College of Computer and Communication Engineering \\ China University of Petroleum \\ Qingdao, China \\ zhangxiaodong@upc.edu.cn
}

\begin{abstract}
In the paper, a cascade predictive control strategy is proposed for the hydraulic AGC (Automatic Gauge Control) system of hot rolling mills based on the data driven control theory to solve the problem of inaccurate indirect measurement and long time-delay direct measurement. Because of the influence of disturbance and uncertainties, press DAGC (Dynamic Automatic Gauge Control) secondary loop control system is added to monitor AGC main loop control system. The proposed method can improve the control precision and ability of disturbance rejection. Simulation results show that the control strategy is effective and has a better control performance.
\end{abstract}

Keywords- data driven; predictive control; automatic gauge control

\section{INTRODUCTION}

Automatic gauge control of hot rolling mills is completed by multi stands. The gauge control of hot strip finishing mills is an important process of the iron and steel industry, and the quality and qualified rate are decided by automatic control level directly. The highly-complex control system presents a challenge for the controller design, which is exacerbated by the hostile hot metal rolling environment. Various control strategy has been used in the field to improve control performance ${ }^{[1-2]}$. To meet the requirement of the automatic gauge control system and improve the product yield, the generic solution of the steady state error in the spring equation was provided in [3]. Limitations of traditional AGC were analyzed, based on the spring equation, and an H-infinity filter was introduced into the forward-feedback loop of AGC to reject the disturbances ${ }^{[4]}$. However, to improve control precision both press AGC control and feedforward AGC control need high precision measured value. In [5], a cascade control scheme was proposed for the AGC system based on the smith prediction control model. A practical active disturbance rejection control (ADRC) solution was proposed for the monitoring automatic gauge control (AGC) system with large timedelay in the hot strip mills ${ }^{[6]}$. Employing a linear reducedorder model with optimized parameters, the practical ADRC was simple to use, easy to tune and energy efficient in dealing with the uncertainties and disturbances.

In the paper, a cascade control scheme is proposed for the AGC system based on the data driven control theory to

\author{
Weixi Gao \\ Beijing Institute of Automatic Control Equipment. \\ Beijing China \\ gaoweixi0811@163.com
}

improve control precision and robust. The paper is organized as follows. Section 2 presents a description of the automatic gauge control system of hot strip mills, and cascade control strategy is presented, where both monitor automatic AGC and press DAGC control system are used. Based on predictive control theory a data driven controller is designed using subsystem identification method in section 3 . In section 4, we present some simulation results to demonstrate that the proposed control method is effect and feasible. Conclusions and future work are given in section 5.

\section{DESCRIPTION OF AGC SYSTEM}

Because we can't get actual thickness of the steel between the roll gaps, press AGC is used widely. Using rolling force and gauge value detected directly, we can calculate the thickness of steel in time with the spring equation. However, the product can't meet the requirement due to its poor accuracy, because of effects of parameter uncertainty and disturbance such as roll eccentricity and oil film thickness changes, etc. So monitor AGC control strategy is used to feedback actual thickness value with detectors fixed after the rear finishing stands about 4-6 meters away. So the actual thickness data can't be used in time and the influence of the time-delay is obviously.

In the paper, both DAGC and monitor AGC are used to improve control performance, where the DAGC secondary loop control system is added to the monitor AGC system, as shown in fig. 1 .

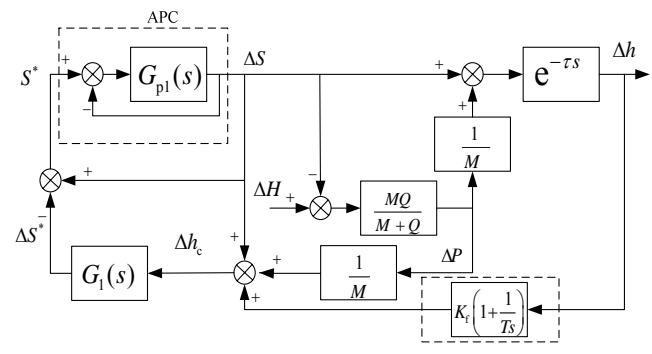

Fig.1 Block diagram of DAGC and monitor AGC

Where $G_{\mathrm{p} 1}(s)$ is the integration transfer function of automatic position control system; $G_{1}(s)$ is controller 
designed for monitor AGC; $K_{\mathrm{f}}\left(1+\frac{1}{T s}\right)$ is the transfer function of monitor $\mathrm{AGC} ; \Delta S, \Delta P$ and $\Delta h$ is increment of gauge, press and thickness, respectively; $\Delta S^{*}$ is gauge increment for $\Delta h ; M$ and $Q$ is stiffness coefficient of rolling mill and plasticity coefficient of steel; $\mathrm{e}^{-\tau \mathrm{s}}$ is time delay part.

From fig. 1, we can see that it is a cascade control system and we can design controller for DAGC system and monitor AGC system, respectively. As shown in fig. 2 a data driven controller is designed for the monitor AGC main loop control system, and controller can be designed directly based on input and output data, and the process of model identification is avoided. The PID control strategy is applied to the secondary loop control system. With the secondary loop control system, disturbances added in the secondary loop are rejected in advance, and the influence of disturbances on the main control loop is weakened.

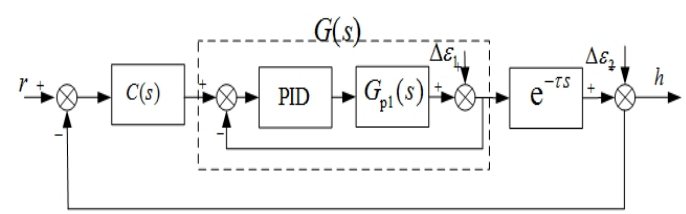

Fig.2 Block diagram of AGC cascade control system

Where $G(s)$ is press AGC control system; $r$ is reference input; $\Delta \varepsilon_{1}, \Delta \varepsilon_{2}$ is external disturbance; $C(s)$ is controller for monitor AGC system.

\section{DATA DRIVEN CONTROLLER DESIGNING}

\section{A. Subspace Identification}

A liner time invariant state space system can be described as ${ }^{[8]}$ :

$$
\begin{aligned}
& \boldsymbol{x}(k+1)=\boldsymbol{A} \boldsymbol{x}(k)+\boldsymbol{B} \boldsymbol{u}(k)+\boldsymbol{K} \boldsymbol{v}(k) \\
& \boldsymbol{y}(k)=\boldsymbol{C} \boldsymbol{x}(k)+\boldsymbol{D} \boldsymbol{u}(k)+\boldsymbol{v}(k)
\end{aligned}
$$

Where $\boldsymbol{u}(k), \boldsymbol{y}(k)$ and $\boldsymbol{x}(k)$ are the process inputs, outputs and states, respectively; $\boldsymbol{A}, \boldsymbol{B}, \boldsymbol{C}, \boldsymbol{D}$ and $\boldsymbol{K}$ are appropriate dimension matrices; $v(k)$ is white noise sequence. We assumed that $(\boldsymbol{A}, \boldsymbol{B})$ is controllable and $(\boldsymbol{A}, \boldsymbol{C})$ is observable, it can be obtained by recursive substation of (1) into (2)

$$
\boldsymbol{Y}_{\mathrm{f}}=\Gamma \boldsymbol{X}_{\mathrm{f}}+\boldsymbol{H}_{\mathrm{f}} \boldsymbol{U}_{\mathrm{f}} \text { or } \boldsymbol{Y}_{\mathrm{p}}=\Gamma \boldsymbol{X}_{\mathrm{p}}+\boldsymbol{H}_{\mathrm{p}} \boldsymbol{U}_{\mathrm{p}}
$$

Where the subscript $\mathrm{p}$ and $\mathrm{f}$ denote "past" and "future". The measurements of the inputs and outputs $\boldsymbol{u}(k)$ and $\boldsymbol{y}(k)$ for $k \in\{0,1, \cdots, 2 N+j-2\}$ are available. The data block Hankel matrices for $\boldsymbol{u}(k)$ represented as $\boldsymbol{U}_{\mathrm{p}}$ and $\boldsymbol{U}_{\mathrm{f}}$ with i-block rows and j-blocks columns are defined as

$$
\begin{gathered}
\boldsymbol{U}_{\mathrm{p}}=\left(\begin{array}{cccc}
\boldsymbol{u}(1) & \boldsymbol{u}(2) & \cdots & \boldsymbol{u}(j) \\
\boldsymbol{u}(2) & \boldsymbol{u}(3) & & \boldsymbol{u}(j+1) \\
\vdots & \vdots & \ddots & \vdots \\
\boldsymbol{u}(N-1) & \boldsymbol{u}(N) & \cdots & \boldsymbol{u}(N+j-1)
\end{array}\right) \\
\boldsymbol{U}_{\mathrm{f}}=\left(\begin{array}{cccc}
\boldsymbol{u}(N) & \boldsymbol{u}(N+1) & \cdots & \boldsymbol{u}(N+j) \\
\boldsymbol{u}(N+1) & \boldsymbol{u}(N+2) & & \boldsymbol{u}(N+j+1) \\
\vdots & \vdots & \ddots & \vdots \\
\boldsymbol{u}(2 N-1) & \boldsymbol{u}(2 N) & \cdots & \boldsymbol{u}(2 N+j-1)
\end{array}\right)
\end{gathered}
$$

Where the outputs block Hankel matrices $\boldsymbol{Y}_{\mathrm{f}}$ and $\boldsymbol{Y}_{\mathrm{p}}$ are defined in the same way.

In the case when no noise is present, the actual future outputs $\boldsymbol{Y}_{\mathrm{f}}$ lies in the combined row space and the linear predictor equation can be written as

$$
\hat{\boldsymbol{Y}}_{\mathrm{f}}=\boldsymbol{L}_{w} \boldsymbol{W}_{\mathrm{p}}+\boldsymbol{L}_{u} \boldsymbol{U}_{\mathrm{f}}
$$

Where $\boldsymbol{W}_{\mathrm{p}}=\left[\begin{array}{ll}\boldsymbol{U}_{\mathrm{p}}^{\mathrm{T}} & \boldsymbol{Y}_{\mathrm{p}}^{\mathrm{T}}\end{array}\right]^{\mathrm{T}}$ and $\boldsymbol{U}_{\mathrm{f}}$ are the past inputs and outputs and future inputs, respectively, $\boldsymbol{L}_{w}$ and $\boldsymbol{L}_{u}$ are subspace matrices corresponding to the states and inputs. By solving the following least-square problem, the outputs prediction $\hat{\boldsymbol{Y}}_{\mathrm{f}}$ can be extracted

$$
\min \left\|\boldsymbol{Y}_{\mathrm{f}}-\left(\boldsymbol{L}_{w}, \boldsymbol{L}_{u}\right)\left(\begin{array}{l}
\boldsymbol{W}_{p} \\
\boldsymbol{U}_{f}
\end{array}\right)\right\|_{F}^{2}
$$

The orthogonal projection of the row space of $\boldsymbol{Y}_{f}$ into the row $W_{p}$ and $U_{f}$, and $\boldsymbol{L}_{w}, \boldsymbol{L}_{u}$ can be obtained via $\mathrm{QR}$ decomposition.

$$
\begin{aligned}
& {\left[\begin{array}{c}
\boldsymbol{W}_{\mathrm{p}} \\
\boldsymbol{U}_{\mathrm{f}} \\
\boldsymbol{Y}_{\mathrm{f}}
\end{array}\right]=\boldsymbol{R} \boldsymbol{Q}=\left[\begin{array}{ccc}
\boldsymbol{R}_{11} & 0 & 0 \\
\boldsymbol{R}_{21} & \boldsymbol{R}_{22} & 0 \\
\boldsymbol{R}_{31} & \boldsymbol{R}_{32} & \boldsymbol{R}_{33}
\end{array}\right]\left[\begin{array}{c}
\boldsymbol{Q}_{1}^{\mathrm{T}} \\
\boldsymbol{Q}_{2}^{\mathrm{T}} \\
\boldsymbol{Q}_{3}^{\mathrm{T}}
\end{array}\right]} \\
& \boldsymbol{L}=\left[\begin{array}{ll}
\boldsymbol{L}_{w} & \boldsymbol{L}_{u}
\end{array}\right]=\left[\begin{array}{ll}
\boldsymbol{R}_{31} & \boldsymbol{R}_{32}
\end{array}\right]\left[\begin{array}{cc}
\boldsymbol{R}_{11} & 0 \\
\boldsymbol{R}_{21} & \boldsymbol{R}_{22}
\end{array}\right]^{\dagger}
\end{aligned}
$$

where “ $†$ " denote the Moore-Penrose pseudo-inverse.

\section{B. Controller Designing}

For the time instant $k$, using the future inputs $\boldsymbol{u}_{f}=\left[\boldsymbol{u}_{k}, \cdots, \boldsymbol{u}_{k+N-1}\right], \boldsymbol{u}_{p}=\left[\boldsymbol{u}_{k-N}, \cdots, \boldsymbol{u}_{k-1}\right]$ and past outputs $\boldsymbol{y}_{p}=\left[y_{k-N}, \cdots, y_{k-1}\right]$, we can get the future 
outputs $\hat{\boldsymbol{y}}_{f}=\left[\hat{\boldsymbol{y}}_{k}, \cdots, \hat{\boldsymbol{y}}_{k+N-1}\right]$, and the prediction expressions of the outputs can be written as

$$
\hat{\boldsymbol{y}}_{f}=\boldsymbol{L}_{w} \boldsymbol{w}_{P}+\boldsymbol{L}_{u} \boldsymbol{u}_{f}
$$

That is

$$
\left[\begin{array}{c}
\hat{\boldsymbol{y}}(k) \\
\hat{\boldsymbol{y}}(k+1) \\
\vdots \\
\hat{\boldsymbol{y}}(k+N-1)
\end{array}\right]=\boldsymbol{L}_{w}\left[\begin{array}{c}
\boldsymbol{u}(k-N) \\
\boldsymbol{u}(k-N+1) \\
\vdots \\
\boldsymbol{u}(k-1) \\
\boldsymbol{y}(k-N) \\
\boldsymbol{y}(k-N+1) \\
\vdots \\
\boldsymbol{y}(k-1)
\end{array}\right]+\boldsymbol{L}_{u}\left[\begin{array}{c}
\boldsymbol{u}(k) \\
\boldsymbol{u}(k+1) \\
\vdots \\
\mathbf{u}(k+N-1)
\end{array}\right]
$$

The cost function to be minimized becomes

$$
\begin{aligned}
J= & \sum_{i=1}^{H_{p}}(\boldsymbol{r}(k+i)-\hat{\boldsymbol{y}}(k+i))^{\mathrm{T}} \boldsymbol{Q}_{i}(\boldsymbol{r}(k+i)-\hat{\boldsymbol{y}}(k+i)) \\
& +\sum_{i=0}^{H_{c}-1} \boldsymbol{u}(k+i)^{\mathrm{T}} \boldsymbol{R} \boldsymbol{u}(k+i) \\
= & \left(\boldsymbol{r}_{f}-\hat{\boldsymbol{y}}_{f}\right)^{\mathrm{T}} \boldsymbol{Q}\left(\boldsymbol{r}_{f}-\hat{\boldsymbol{y}}_{f}\right)+\boldsymbol{u}_{f}^{\mathrm{T}} \boldsymbol{R} \boldsymbol{u}_{f}
\end{aligned}
$$

Where $H_{p}$ and $H_{c}$ denote the predictive horizon and control horizon, respectively. $\boldsymbol{r}$ are reference inputs. The output and input weighting matrices $\boldsymbol{Q}=\operatorname{diag}\left(\boldsymbol{Q}_{1} \cdots \boldsymbol{Q}_{H_{p}}\right)>0$ and $\boldsymbol{R}=\operatorname{diag}\left(\begin{array}{lll}\boldsymbol{R}_{1} & \cdots & \boldsymbol{R}_{\boldsymbol{H}_{c}}\end{array}\right) \geq 0$.To find the minimum of $J$, its derivative is set to zero

$$
\frac{\partial J}{\partial \boldsymbol{u}_{f}}=0
$$

The control law are therefore defined as

$$
\boldsymbol{u}(k)=\left(\boldsymbol{R}+\boldsymbol{L}_{u}^{\mathrm{T}} \boldsymbol{Q} \boldsymbol{L}_{u}\right)^{-1} \boldsymbol{L}_{u}^{\mathrm{T}} \boldsymbol{Q}\left(\boldsymbol{r}-\boldsymbol{L}_{w} \boldsymbol{W}_{p}\right)
$$

and only the first control law is used to the system at time $k$.

$$
\begin{aligned}
\boldsymbol{u}(k)= & \left(\begin{array}{llll}
\boldsymbol{I} & 0 & \cdots & 0
\end{array}\right) \cdot \\
& \left(\boldsymbol{R}+\boldsymbol{L}_{u}^{\mathrm{T}} \boldsymbol{Q} \boldsymbol{L}_{u}\right)^{-1} \boldsymbol{L}_{u}^{\mathrm{T}} \boldsymbol{Q}\left(\boldsymbol{r}-\boldsymbol{L}_{w} \boldsymbol{W}_{p}\right)
\end{aligned}
$$

\section{SIMULATION RESULTS}

In this section, we will use the simplified second order transfer function from the engineering practice to illustrate the performance of the proposed control strategy.

$$
G(s)=\frac{2110.7}{s^{2}+1983.7 s+39.7}
$$

We set control horizon $H_{c}=2$; predictive horizon $H_{P}=4$; sampling time is $5 \mathrm{~ms}$; and input disturbance $\Delta \varepsilon_{1}=0.08 \sin (12 t+90)+0.04 \cos (24 t)+0.008$ as shown in fig. 3 .

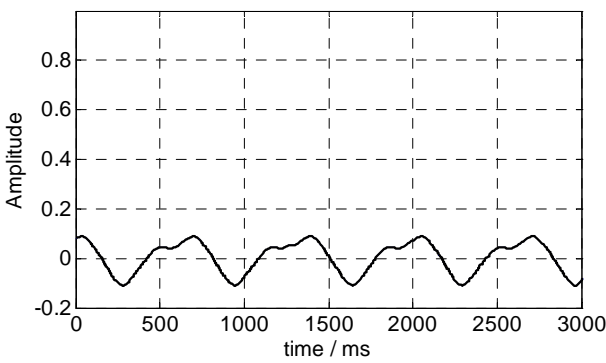

Fig. 3 Input of disturbance

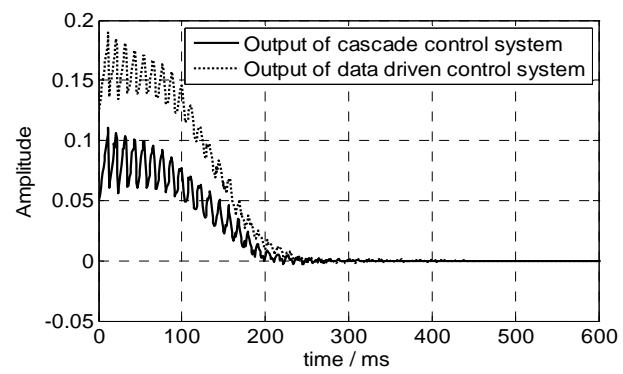

Fig. 4 Output of the system

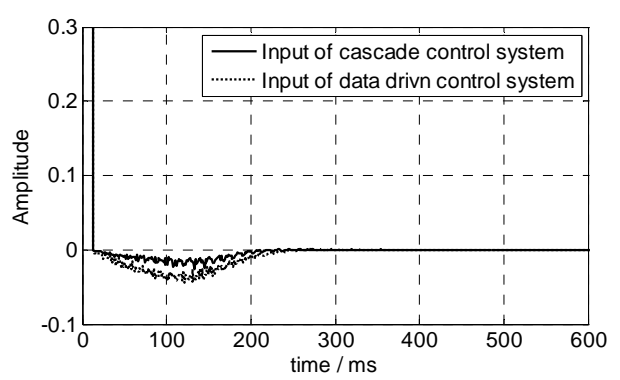

Fig. 5 Output of the system with disturbance

Fig. 4 compares the outputs of the control system of two control strategy. it can be seen that, we can get better control effects using cascade control strategy compared with data driven control strategy alone, where second looper PID control, we set parameter values $\mathrm{P}=80, \mathrm{I}=0.4$. In addition, we can improve performance by setting different value of PID parameter. Fig. 5 compares inputs of control system of two control strategy. In summary, the disturbance rejection and control precision can be improved using cascade control strategy.

\section{CONCLUSIONS}

In the paper, we design cascade control strategy for automatic gauge control system, where we design data driven controller for main loop monitor AGC system as well as PID controller for secondary loop DAGC system. The PID second loop control system can reject disturbance in advance, and reacts quickly and able to compensate the disturbance faster compared to the data driven control system. Furthermore, based on data driven control theory, the model identification process is avoided and control precision and performance is improved. Simulation results 
show the efficiency and feasibility of the proposed control strategy.

\section{ACKNOWLEDGMENT}

This work is funded by research grants from the Shandong Provincial Natural Science Foundation, China (No. ZR2012FL16 and ZR2011FQ018) the National Natural Science Foundation of China (No. 61305008), and the Fundamental Research Funds for the Central Universities (No. 14CX02029A and 11CX04054A).

\section{REFERENCES}

[1] Hearns G, Grimble M J. Robust multivariable control for hot strip mills. ISIJ Int, 2000, 40(10): 995-999.

[2] Fu J, Yang W D, Li B Q, et al. Robust control for dynamic set AGC in hot strip mill based on $\mu$ synthesis. Journal of University of Science and Technology Beijing, 2006, 28(3): 293-298

[3] Peng Wen Zhang Dianhua Cao Jianzhao, Liu Ziying. Optimization Algorithm of Spring Equation in Tandem Hot Rolling Based on Static Errors [J]. Journal of northeastern university (natural science edition), 2013 (4):528-531.

[4] Robust predictive-model-control strategy for automatic gauge control in hot strip finishing milling [J]. Control theory and applications, 2011, 28 (6):799-804.

[5] ZHANG Xiao-dong, YAO Xiao-lan, WU Qing-he, XIN Qiu-li. Cascade predictive control for AGC in hot strip rolling. Journal of University of Science and Technology Beijing, 2011,33 (8):1013-1017.

[6] WANG Li-jun, TONG Chao-nan, LI Qing1,YIN Yi-xin, GAO Zhi-qiang, ZHENG Qin-ling. Practical active disturbance rejection solution for monitoring automatic gauge control system with large time-delay [J]. Control theory and applications. 2012,29(3): 368 -374.

[7] YANG Hua, LI Shao-yuan. A novel robust predictive controller design based on data-driven subspace identification. Control theory and Applications, 2007, 24(5): 732-736.

[8] N.A. Wahab, R, Katebi, J. Balderud, M.F. Rahmat. Datadriven adaptive model-based predictive control with application in wastewater systems. IET control theory appl. 2011,5(6):803-812 